\title{
Electron cloud driven vacuum instability
}

\author{
W. Fischer, U. Iriso, and E. Mustafin \\ To be submitted to the proceedings of the \\ $33^{\text {rd }}$ ICFA Beam Dynamics Workshop on High Intensity and High Brightness Beams, \\ Bensheim, Germany, October 2004
}

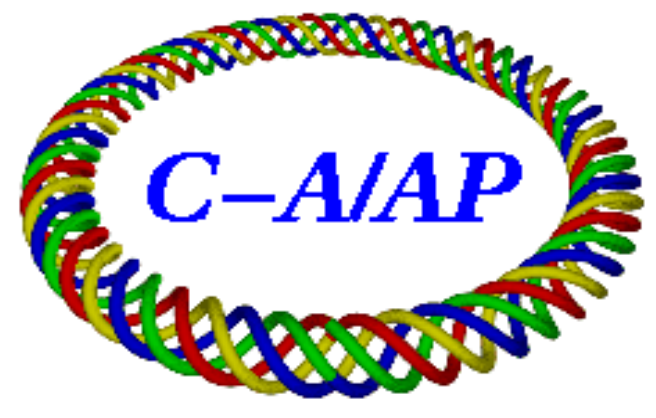

Collider-Accelerator Department Brookhaven National Laboratory

Upton, NY 11973 


\title{
Electron cloud driven vacuum instability ${ }^{12}$
}

\author{
W. Fischer*, U. Iriso* and E. Mustafin ${ }^{\dagger}$ \\ * Brookhaven National Laboratory, Upton, NY 11973, USA \\ ${ }^{\dagger}$ Gesellschaft für Schwerionenforschung, Darmstadt, Germany
}

\begin{abstract}
Electron clouds are shown to cause pressure rises in the Relativistic Heavy Ion Collider. In a number of cases beam induced vacuum instabilities were seen where the pressure grows exponentially with time. We analyze under which conditions electron clouds can lead to these vacuum instabilities. We consider as the feedback mechanism for the instability the following: rest gas ionization by electrons in the cloud and the beam, subsequent acceleration of the ions by the beam, and molecular desorption induced by the ions hitting the wall, leading to increased pressure and thus higher ionization rates.
\end{abstract}

\section{INTRODUCTION}

The Relativistic Heavy Ion Collider (RHIC) consists of two superconducting rings, named Blue and Yellow. Since 2001 vacuum pressure rises were observed with intense ion beams. While this could be seen initially only at injection, later observations were also made at store and at transition. Pressure rises were observed with all species $\left(\mathrm{Au}^{79+}, \mathrm{d}^{+}, \mathrm{p}^{+}\right)$, and with two exceptions, only in the warm interaction regions. A summary of the observations, and further references can be found in [1].

A number of effects were considered to account for the observed pressure rises [2]. The existence of electron clouds in conjunction with pressure rises could be confirmed by observing the tune shift in bunch trains [3], and by direct observation with electron detectors [4]. The ionization of rest gas by the beam, subsequent acceleration of the ions in the beam field, and the desorption when the ions hit the wall, is only a possibility for ions with high charge states. The contributions of beam losses are still under investigation, and anti-grazing rings were proposed for mitigation [5].

In a number of cases pressures growing exponentially with time were observed (see Fig. 1 for an example). Here we analyze under which conditions electron clouds can lead to these vacuum instabilities.

\section{OBSERVATIONS}

Fig. 1 shows an example of a pressure rise instability in the Blue ring that limited the beam intensity during the 2004 operating period. The pressure rise occurred in the collimator region that was not baked due to scheduling conflicts. The Yellow collimators were baked, and no

\footnotetext{
1 Work performed under the auspices of the US Department of Energy. 2 At the workshop the author presented the overview talk "Electron clouds and vacuum pressure rise in RHIC". Most of the material in the talk is documented in Ref. [1]. Here an more detailed analysis of a particular aspect is presented that was not published previously.
}

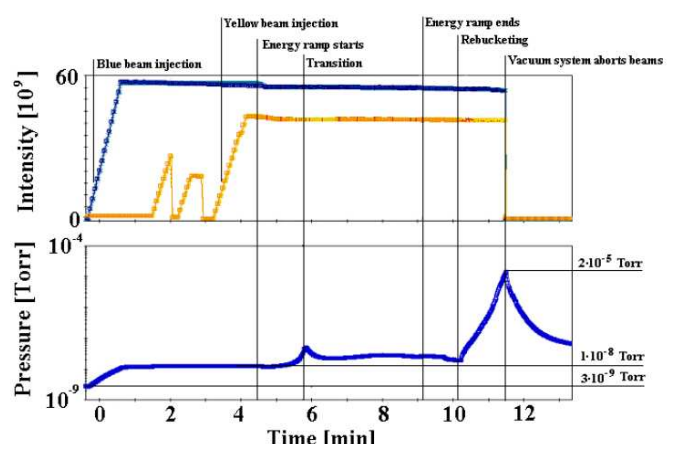

FIGURE 1. A vacuum instability with Au beam in the Blue ring. The upper part shows the total intensity for both rings during injection, acceleration, and storage. The lower part shows the pressure in the Blue collimator region, with an exponential increase after rebucketing.

vacuum instabilities were observed there.

The Blue ring is filled first, resulting in a slight pressure increase. After the Yellow beam is filled both beams are accelerated. A pressure increase is visible when transition is crossed, as the bunches get shorter. The pressure drops back after the transition crossing. When the bunches reach the flattop energy, they are transferred from the accelerating rf system with harmonic number 360 into the storage rf system with harmonic number 2520 . In the process, the bunch length is reduced by about 50\%. After rebucketing, the pressure increases exponential with a time constant of 11.7 seconds until the vacuum interlock system aborts the beams. It is likely that the bunch shortening triggered the formation of an electron cloud. This has also been seen at another location in the ring [6]. No electron detectors are installed in the collimator region. The beam parameters are listed in Tab. 1. A second pressure instability of the same type was observed with 61 bunches of $0.9 \cdot 10^{9}$ average intensity, with a growth time of 5.9 seconds. For operation the beam intensity was kept below the instability threshold.

To clarify the role of electron clouds and beam loss 2 tests were made. First, Au bunches were injected with 
TABLE 1. Input parameters, computed $\eta_{\text {crit, ion }}$ for $H_{2}$ and $C O$, and $\eta_{i o n}$ calculated from growth times for the unbaked Blue collimator region. The parameters for gold correspond to the vacuum instability shown in Fig. 1. The value for protons show the highest injected proton intensity, for which no instability developed.

\begin{tabular}{|c|c|c|c|}
\hline parameter & unit & $\mathrm{Au}^{79+}$ & $\mathrm{p}^{+}$ \\
\hline $\begin{array}{l}\text { beam parameters } \\
\text { charge, mass number } Z, A \\
\text { relativistic } \gamma \\
\text { particles per bunch } N_{a} \\
\text { no of bunches } N_{b}\end{array}$ & $\begin{array}{c}\cdots \\
\ldots \\
10^{9} \\
\ldots\end{array}$ & $\begin{array}{c}79,197 \\
107.4 \\
1 \\
56\end{array}$ & $\begin{array}{l}1,1 \\
25.9 \\
170 \\
111\end{array}$ \\
\hline $\begin{array}{l}\text { vacuum system } \\
\text { pressure } P_{0} \\
\text { temperature } T \\
\text { pipe radius } r \\
\text { conductance } c_{H 2} \\
\text { conductance } c_{C O} \\
\text { space betw. pumps } 2 L\end{array}$ & $\begin{array}{l}\text { Torr } \\
\mathrm{K} \\
\mathrm{m} \\
\mathrm{m}^{4} \mathrm{~s}^{-} \\
\mathrm{m}^{4} \mathrm{~s}^{-} \\
\mathrm{m}\end{array}$ & \multicolumn{2}{|c|}{$\begin{array}{c}1.0 \cdot 10^{-8} \\
300 \\
0.08 \\
0.56 \\
0.15 \\
11.4\end{array}$} \\
\hline $\begin{array}{l}\text { ionization by cloud electror } \\
\text { current into wall } d I_{e} / d l \\
\text { average electron energy } \\
\text { cross section } \sigma_{e, H 2} \\
\text { cross section } \sigma_{e, C O}\end{array}$ & $\begin{array}{c}{[3,4,} \\
\mathrm{A} / \mathrm{m} \\
\mathrm{eV} \\
\mathrm{m}^{2} \\
\mathrm{~m}^{2}\end{array}$ & \multicolumn{2}{|c|}{$\begin{array}{c}0.03 \\
50 \\
9.8 \cdot 10^{-21} \\
2.2 \cdot 10^{-20}\end{array}$} \\
\hline $\begin{array}{l}\text { ionization by beam }[8,10] \\
\text { cross section } \sigma_{b, H 2} \\
\text { cross section } \sigma_{b, C O}\end{array}$ & $\begin{array}{l}\mathrm{m}^{2} \\
\mathrm{~m}^{2}\end{array}$ & $\begin{array}{l}1.3 \cdot 10^{-19} \\
5.8 \cdot 10^{-19}\end{array}$ & $\begin{array}{l}2.2 \cdot 10^{-23} \\
1.0 \cdot 10^{-22}\end{array}$ \\
\hline $\begin{array}{l}\text { ion energy at wall } \\
\text { reported } \eta_{H 2}[8] \\
\text { reported } \eta_{C O}[8] \\
\text { desorp. coeff. } \eta_{c r i t, H 2} \\
\text { desorp. coeff. } \eta_{c r i t, C O}\end{array}$ & $\begin{array}{l}\mathrm{eV} \\
\cdots \\
\cdots \\
\cdots \\
\cdots\end{array}$ & $\begin{array}{l}15 \\
0.4 \\
0.3 \\
42 \\
2.8\end{array}$ & $\begin{array}{c}62 \\
1.5 \\
1.2 \\
131 \\
14\end{array}$ \\
\hline $\begin{array}{l}\text { growth time meas. } \tau \\
\text { corresponding } \eta_{H 2} \\
\text { corresponding } \eta_{C O}\end{array}$ & $\begin{array}{l}\mathrm{s} \\
\mathrm{s} \\
\mathrm{s}\end{array}$ & $\begin{array}{c}6-12 \\
23-21 \\
2.1-1.7\end{array}$ & \\
\hline
\end{tabular}

different spacings. Second, a local beam loss was created by moving in one of the Blue collimators. In the first test 53 bunches with 108 ns spacing and $10^{9}$ bunch intensity lead to a pressure of $7 \cdot 10^{-6}$ Torr. Injection of approximately the same amount of beam with twice the bunch spacing lead to a pressure of only $4 \cdot 10^{-8}$ Torr. In the second test a local beam loss of $7 \cdot 10^{7} \mathrm{Au}$ ions within 5 second did not induce any pressure rise. Thus the pressure rise in the collimator region is sensitive to bunch length and bunch spacing, but not to local beam losses. This is consistent with electron clouds as the mechanism driving the pressure rise.

Before 2004, exponential pressure rises were also seen at injection, with growth times ranging from 1.7 to 7.1 second. In all these cases Au beam was injected, and the pressure rise occurred in unbaked regions.

\section{ANALYSIS}

To describe the pressure evolution $P$ we consider a model that includes a static gas load $Q_{0}$, a load $Q_{1}$ from elec-

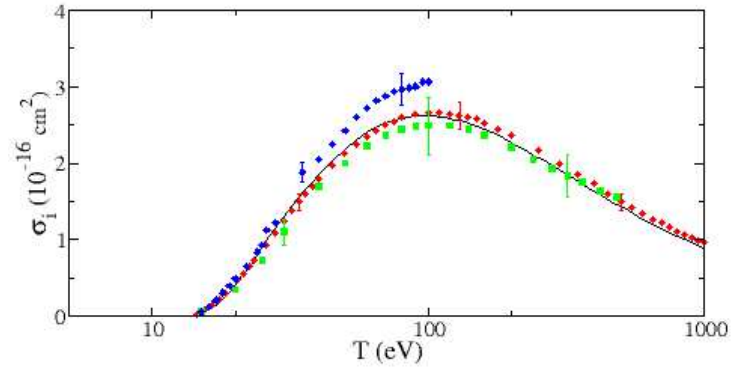

FIGURE 2. Ionization cross section of $\mathrm{CO}$ molecules as a function of the electron impact energy [7]. The solid line shows the output of a model, the colored points denote 3 different measurements.

trons in a cloud hitting the walls, a load $Q_{2}$ from rest gas molecules ionized by the cloud electrons and accelerated by the beam, and a load $Q_{3}$ from rest gas molecules ionized and accelerated by the beam. The total load is then

$$
Q=Q_{0}+Q_{1}+Q_{2}+Q_{3} \text {. }
$$

We will derive a criterion for vacuum stability, and estimate the growth time in an unstable situation. We compare expectations from the model with observations.

We model the collimator region as a periodic structure with pumps of pumping speed $2 S$ spaced by the distance $2 L$. The three pumps in the region are spaced by $9.2 \mathrm{~m}$ and $11.4 \mathrm{~m}$, and the conductance $c$ varies due to the insertion of the collimators. We take limiting values for $2 L$ and $c$ (see Tab. 1) for the calculations.

\section{Stability criterion}

We consider the equilibrium $Q=S P$. The load $Q_{1}$ per length $L$ is

$$
Q_{1}=k T \frac{L}{e} \frac{d I_{e}}{d l} \eta_{e}
$$

where $k$ is the Boltzmann constant, $T$ the absolute temperature, $e$ the elementary charge, and $d I_{e} / d l$ the electron current into the wall per unit length. $\eta_{e}$ is the average desorption coefficient for the energy distribution of the cloud electrons. A pressure change will affect the rate at which rest gas molecules are ionized and electrons generated. However, in simulations the variation of the electron generation rate did not change the electron cloud density nor the time to reach saturation [3]. We therefore assume that the gas load $Q_{1}$ does not depend on the pressure $P$, and thus will not lead to an instability.

The load $Q_{2}$ can be estimated as

$$
Q_{2}=\sigma_{e} P \frac{2 r L}{e} \frac{d I_{e}}{d l} \eta_{i o n}
$$

where $\sigma_{e}$ is the cross section for rest gas ionization from an impact of cloud electrons, $r$ the beam pipe radius, and $\eta_{i o n}$ is the average desorption coefficient for ions accelerated by the beam. Values for $\sigma_{e}$ can be found in [7], an example for $C O$ is shown in Fig. 2. The gas load $Q_{3}$ is [8]

$$
Q_{3}=\sigma_{b} P L \dot{N}_{b} \eta_{\text {ion }}
$$


where $\sigma_{b}$ is the cross section for the rest gas ionization, $\dot{N}_{b}$ is the beam particle flow, i.e. the number of particles in the beam divided by the revolution time. In equilibrium we have $P S=Q$, where $S$ is the pumping speed. Introducing the parameter

$$
b=\sigma_{e} \frac{2 r}{e} \frac{d I_{e}}{d l}+\sigma_{b} \dot{N}
$$

we therefore get for the equilibrium pressure measured at the pump

$$
P=\frac{Q_{0}+k T \frac{L}{e} \frac{d I_{e}}{d l} \eta_{e}}{S-\eta_{\text {ion }} L b}
$$

From Eq. (6) a stability condition can be derived. However, in conductance limited systems a more stringent condition applies and the maximum desorption coefficient becomes [8]

$$
\eta_{\text {crit }, \text { ion }}=\frac{\pi^{2}}{4} \frac{c}{b L^{2}}
$$

\section{Measurement of desorption coefficient}

We will now estimate the ion desorption coefficient $\eta_{i o n}$ from the measured growth time $\tau$. The pressure follows the differential equation [9]

$$
v(x) \frac{\partial P(x, t)}{\partial t}=a(x)+\eta_{\text {ion }} b(x) P(x, t)+c(x) \frac{\partial^{2} P(x, t)}{\partial x^{2}}
$$

where $v(x)$ is the volume per unit length, $a(x)$ the static gas desorption per unit length, and $b(x)$ and $c(x)$ were introduced above. In a situation with exponential pressure rise the static load $a(x)$ can be neglected, and we make the ansatz

$$
P(x, t)=e^{t / \tau} p(x) .
$$

where $\tau$ is the growth time. Introducing the variable

$$
\omega^{2}(x)=\frac{\eta_{\text {ion }} b(x)-v(x) / \tau}{c(x)}
$$

and assuming weak $x$-dependence of $\omega$, Eq. (8) can be written as

$$
\frac{d^{2} p(x)}{d x^{2}}+\omega^{2} p(x)=0
$$

with the general solution $p(x)=A \cos [\omega(x-L)]$, which takes into account the symmetry around $x=L$. From the boundary conditions $\left.c \frac{d P}{d x}\right|_{x=0}=\left.S P\right|_{x=0}$ it follows

$$
(\omega L) \tan (\omega L)=S L / c,
$$

which can only be numerically solved for $\omega$. Denoting the smallest root with $\omega_{0}$ we find

where $r$ is the pipe radius.

$$
\eta_{\text {ion }}=\left[\omega_{0}^{2} c+\pi r^{2} / \tau\right] / b
$$

\section{DISCUSSION}

All input parameters are shown in Tab. 1 as well as the calculated $\eta_{c r i t, i o n}$, and the $\eta_{i o n}$ corresponding to the measured growth time using Eq. (13). Calculations were done for $\mathrm{H}_{2}$ and $\mathrm{CO}$ while cross terms are neglected. The critical desorption coefficients for $\mathrm{H}_{2}$ are large therefore $\mathrm{H}_{2}$ is not likely to play a critical role. The critical desorption coefficient for $C O$ is still considerable. Although the desorption coefficient calculated from the growth time is still somewhat smaller than the calculated critical one, the gap is not very large. We note that Eq. (7) probably overestimates $\eta_{\text {crit,ion }}$ by some $30 \%$ [8]. An instability appears possible for $C O$ like molecules.

To get an estimate for actual desorption coefficients, we compute the energy $E_{i o n}$ of an ion with a single electron charge moving in the beam potential to the wall [11]:

$$
E_{\text {ion }}=\frac{Z e^{2} N_{a}}{2 \pi \varepsilon_{0} L_{\text {sep }}} \ln \left(\frac{r}{\sigma_{r}}\right)
$$

where $\sigma_{r}$ is the rms beam radius, and $L_{\text {sep }}$ the bunch separation. The reported desorption coefficients are still below the critical ones by about an order of magnitude. However, in [8] only an interpolation is given between zero and $500 \mathrm{eV}$, and no measurements exist below $100 \mathrm{eV}$ [12]. We can only speculate that the actual ion desorption coefficients are larger than those reported in [8] or that there is a significant effect from ionization to higher charge states.

Significant uncertainty is also in the properties of the electron cloud. With our parameters, the ionization from the cloud electrons is about $20 \%$ of the beam ionization for $\mathrm{Au}$ ions. For protons the rest gas ionization can be neglected. This explains why no vacuum instability has been observed with protons.

Baking reduces the ion desorption coefficients by up to an order of magnitude [8], and at the same time the secondary electron yield. It should therefore suppress any vacuum instabilities. This is consistent with our observations. While vacuum instabilities can be suppressed, it is still possible to create intolerable pressure rises.

\section{ACKNOWLEDGMENTS}

We are thankful to J. Alessi, M. Blaskiewicz, M. Jimenez, A. Krämer, E. Mahner, H.C. Hseuh, F. Ruggiero, G. Rumolo, P. Thieberger, D. Trbojevic, J. Wei, S.Y. Zhang, and F. Zimmermann for support and discussions.

\section{REFERENCES}

1. W. Fischer et al., ECLOUD'04, Napa, California (2004).

2. W. Fischer et al., "Vacuum pressure rise with intense ion beams in RHIC", EPAC 2002, Paris (2002).

3. W. Fischer, J.M. Brennan, M. Blaskiewicz, T. Satogata, Phys. Rev. ST Accel. Beams 5, 124401 (2002).

4. U. Iriso et al., BNL C-A/AP/129 (2003).

5. P. Thieberger et al., Phys. Rev. ST Accel. Beams 7, 093201 (2004).

6. G. Rumolo and W. Fischer, BNL C-A/AP/146 (2004).

7. Y.-K. Kim et al., NIST physics reference data for electronimpact cross sections for ionization and excitation, http://physics.nist.gov/PhysRefData/Ionization/ (2004).

8. O. Gröbner, in CERN 99-05, p 132 (1999).

9. A.G. Mathewson, CERN 94-01, Vol II, p 717 (1994).

10. F.F. Rieke, W. Prepejchal, Phys. Rev. A 4, p 1507 (1972).

11. G. Rumolo, F. Zimmermann, CERN-SL-2001-014 AP.

12. E. Mahner, private communication (2004). 